
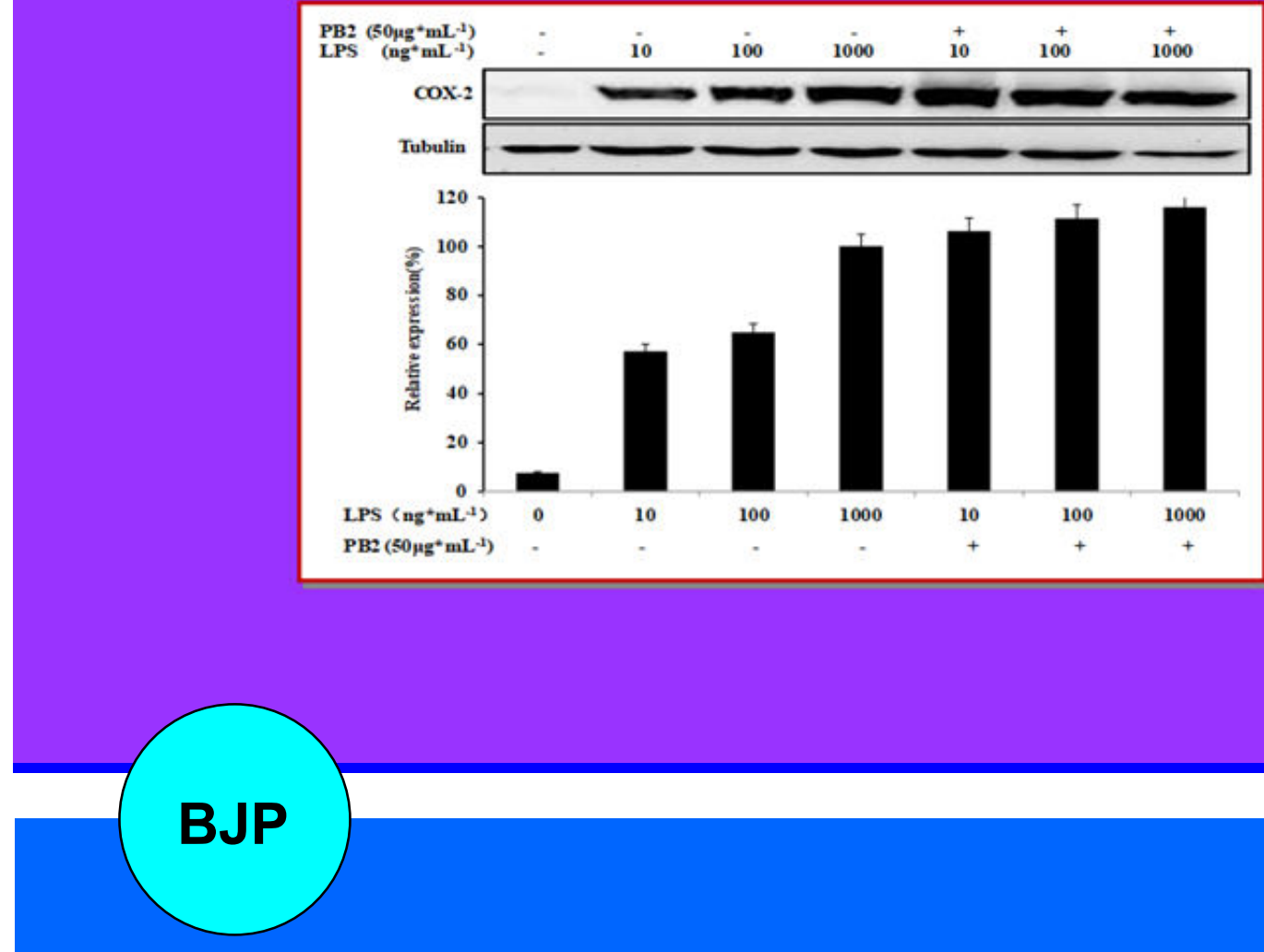

Bangladesh Journal of Pharmacolo-

Research Article

Preparation of procyanidin B2 from apple pomace and its inhibitory effect on the expression of cyclooxygenase-2 in lipopolysaccharidetreated RAW264.7 macrophages 


\title{
Preparation of procyanidin B2 from apple pomace and its inhibitory effect on the expression of cyclooxygenase-2 in lipopolysaccharide -treated RAW264.7 macrophages
}

\author{
Huawei Zhang and Chen Ying
}

Department of Pharmacy, School of Pharmaceutical Sciences, Zhejiang University of Technology, Hangzhou 310 014, China.

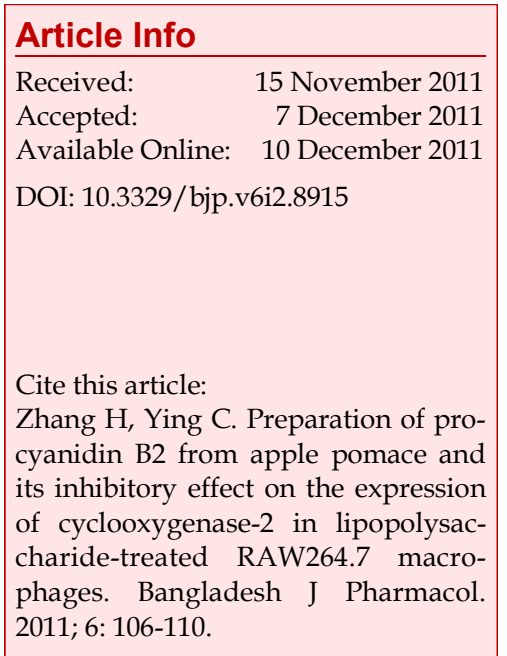

\begin{abstract}
Dimeric procyanidin B2 (PB2) is one of phenolic compounds in apple pomace, an agro-industrial byproduct in apple juice processing. This work focused on purification of PB2 from apple pomace using sephadex column chromatography and its potential effect on lipopolysaccharide (LPS)-induced inflammation using RAW264.7 macrophages. PB2 with the purity of $72.3 \pm$ $1.9 \%$ was successfully afforded using resin and gel column chromatographic technique. Anti-inflammatory tests suggested that the expression of cyclooxygenase-2 (COX-2) in LPS-induced murine RAW264.7 macrophages was suppressed in a PB2 concentration-dependent manner. PB2 at no less than $50 \mu \mathrm{g} \mathrm{mL}-1$ could significantly suppress inflammation in the LPSinduced cells. Moreover, this suppressive effect was not correlated with PB2 pretreating. However, the COX-2 expression was not reduced in LPS pretreatment way followed by PB2 exposure, which suggested that PB2 has no repairing function. The results showed that high pure PB2 prepared from apple pomace has a remarkable anti-inflammatory property.
\end{abstract}

\section{Introduction}

China is one of the largest exporters of concentrated apple juice. However, over 3 million tons of apple pomace is yearly generated from juice processing factory. How to deal with this argo-byproduct has become an important environmental problem. Many papers had reported that apple pomace contains a variety of bioactive compounds including polyphenols, composed of primarily monomeric flavanols (-)-epicatechin and (+)-catechin, and oligomeric procyanidins formed from these monomeric units, such as dimeric procyanidin B2 (PB2) (Figure 1) (García et al., 2009; Suarez et al., 2010; Vendruscolo et al., 2008). Pharmacological tests had shown that PB2 has a broad spectrum of bioactivities, such as anti-inflammation, anti-tumor, anti-oxidant activity (Miura et al., 2008; Sakano et al.,
2005; Zhang et al., 2006). In our previous study, polyphenols-enriched extract from apple pomace was subjected to fractionation using macroporous absorbent resin XAD-16 and one fraction APIII was found to have the highest content of PB2 $(22.5 \pm 1.2 \%)$.

Cyclooxygenase (COX), the key protein that catalyzes the rate-limiting step in prostaglandin biosynthesis, exists in at least two isoforms, designated as COX-1 and 2. Although these proteins share considerable sequence homology, these isoforms differ considerably in the tissue distribution, regulation, and function (Kim et al., 2007; Wu et al., 2010). COX-1 is a housekeeping protein, being constitutively expressed in almost all mammalian tissues. In contrast, COX-2 is barely detectable under normal physiological conditions. However, like other early-response gene products, COX-2 can be induced 


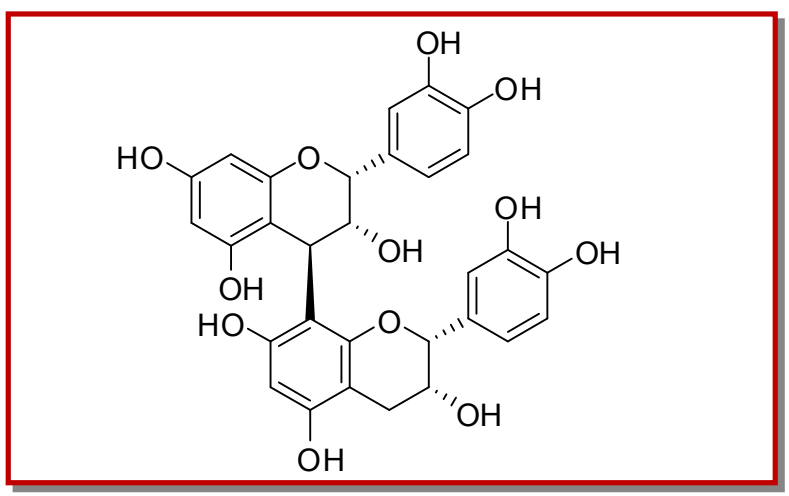

Figure 1: The chemical structure of procyanidin B2 (PB2)

rapidly and transiently by pro-inflammatory mediators and mitogenic stimuli, including cytokines, endotoxins, growth factors, oncogenes, and phorbol esters. So, COX -2 has received considerable attention for its potential role in the inflammation and disease development and is recognized as a potential therapeutic target for the prevention and treatment of a number of diseases (Fujiwara and Kobayashi, 2005).

In this work, we investigated inhibitory effect of PB2 purified from apple pomace on COX-2 expression induced by the endotoxin lipopolysaccharide (LPS) at different concentration and time. Furthermore, the repairing function of PB2 was also studied through reverse pretreatment.

\section{Materials and Methods}

\section{Apple pomace}

Fresh apple pomace was obtained from Shaanxi Hengxing Fruit Juice Co., Ltd, the largest manufacturer of apple juice concentrate in China. About $100 \mathrm{~g}$ dried apple pomace was extracted with aqueous alcohol according to a previous published protocol (Bai et al., 2010). The afforded extract was concentrated under reduced pressure (Eyela NC-2000, Japan) and lyophilized (Freezone 2.5 plus, USA). After resolving in 200 $\mathrm{mL}$ of $20 \%$ ethanol solution, the resulting extract was centrifuged for $15 \mathrm{~min}$ at 10,000 rpm (CR21GII, Hitachi Koki Co., Ltd., Japan), and preserved at $4^{\circ} \mathrm{C}$.

\section{Chemicals}

LPS, procyanidins B2 (purity 98.3\%) and dimethyl sulfoxide (DMSO) were purchased from Sigma-Aldrich Chemical Co. (USA). Fetal bovine serum (FBS) was from HyClone Co., USA. Dulbecco's modified eagle's medium (DMEM), penicillin and streptomycin were obtained from Invitrogen Co., USA. Marker proteins, enhanced chemiluminescence (ECL) and bicinchoninic acid (BCA) protein assay kits were purchased from Pierce Co., USA. Anti-COX-2, anti-a-Tubulin and horse- radish peroxidase-conjugated anti-goat secondary antibody were purchased from Santa Cruz Biotechnology Inc. (USA). Nitrocellulose film was from Bio-rad Co., USA. All others chemicals were of HPLC or analytical grade.

\section{Purification of PB2}

$200 \mathrm{~mL}$ of polyphenol-enriched extract was retained on $100 \mathrm{~mL}$ macroporous adsorbent resin XAD-16 (Rohm and Haas, USA) and packed into a normal atmosphere column $(300 \times 30 \mathrm{~mm})$. The extract were gradually eluted with three bed volumes of distilled water and aqueous alcohol, which concentrations were 20, 40, 60, 80 and $100 \%$, respectively. Six collected fractions, respectively named as API, APII, APIII, APIV, APV and APVI, were concentrated, free-dried and preserved at $18^{\circ} \mathrm{C}$ until further purification with gel filtration. Preliminary HPLC analysis showed that fraction APIII had the highest content of PB2 $(22.5 \pm 1.2 \%)$.

About $1 \mathrm{~g}$ of fraction APIII was dissolved in $4 \mathrm{~mL}$ enthanol and centrifuged at 10,000 rpm for $10 \mathrm{~min}$. Then the supernatant was subjected to gel filtration on a glass column $(600 \times 30 \mathrm{~mm})$ with Sephadex LH-20 (Phamacia Co., Sweden). During the purification process, one subfraction was collected and lyophilized. HPLC analysis of the subfraction was carried out on an Agilent 1200 series liquid chromatograph (Agilent Co., USA)/a diode array detector equipped with a reversedphase Sunfire C8 $(250 \times 4.6 \mathrm{~mm}$ ID, $5 \mu \mathrm{m})$ (Waters Co., USA). Wavelength monitoring was performed at 280 $\mathrm{nm}$. The eluting solvents consisted of $70 \%$ aqueous $0.1 \%$ acetic acid (solvent A) and 30\%acetonitrile (solvent B). All solvents were filtered with a $0.5 \mu \mathrm{m}$ membrane filter before HPLC analysis. Column oven and flow-rate were respectively set at $25^{\circ} \mathrm{C}, 1.0 \mathrm{~mL} / \mathrm{min}$, and the injection volume was $10 \mu \mathrm{L}$. Quantification of PB2 was done by the external standard method.

\section{Cell culture}

The mouse macrophage cell line RAW 264.7 was obtained from Cell Storehouse of Chinese Academy of Science (Shanghai, China) and grown in Dulbecco's modified Eagle's medium supplemented with $10 \%$ fetal bovine serum and 100 units/mL penicillin/streptomycin sulfate. The cells incubated in a humidified incubator with $5 \% \mathrm{CO}_{2}$ atmosphere at $37^{\circ} \mathrm{C}$ were fed with fresh medium every 2 days and subcultured when they reached a confluence of $80 \%$.

\section{COX-2 proteins assay}

Murine RAW 264.7 macrophage cells were grown and subcultured when they reached a confluence of $80 \%$. Testing samples were prepared using preparative PB2 and DMSO, including 1, 10, 50 and $100 \mu \mathrm{g} / \mathrm{mL}$. In order to assay anti-inflammatory activity, the cells were pretreated with different concentration PB2 for 4 hours 


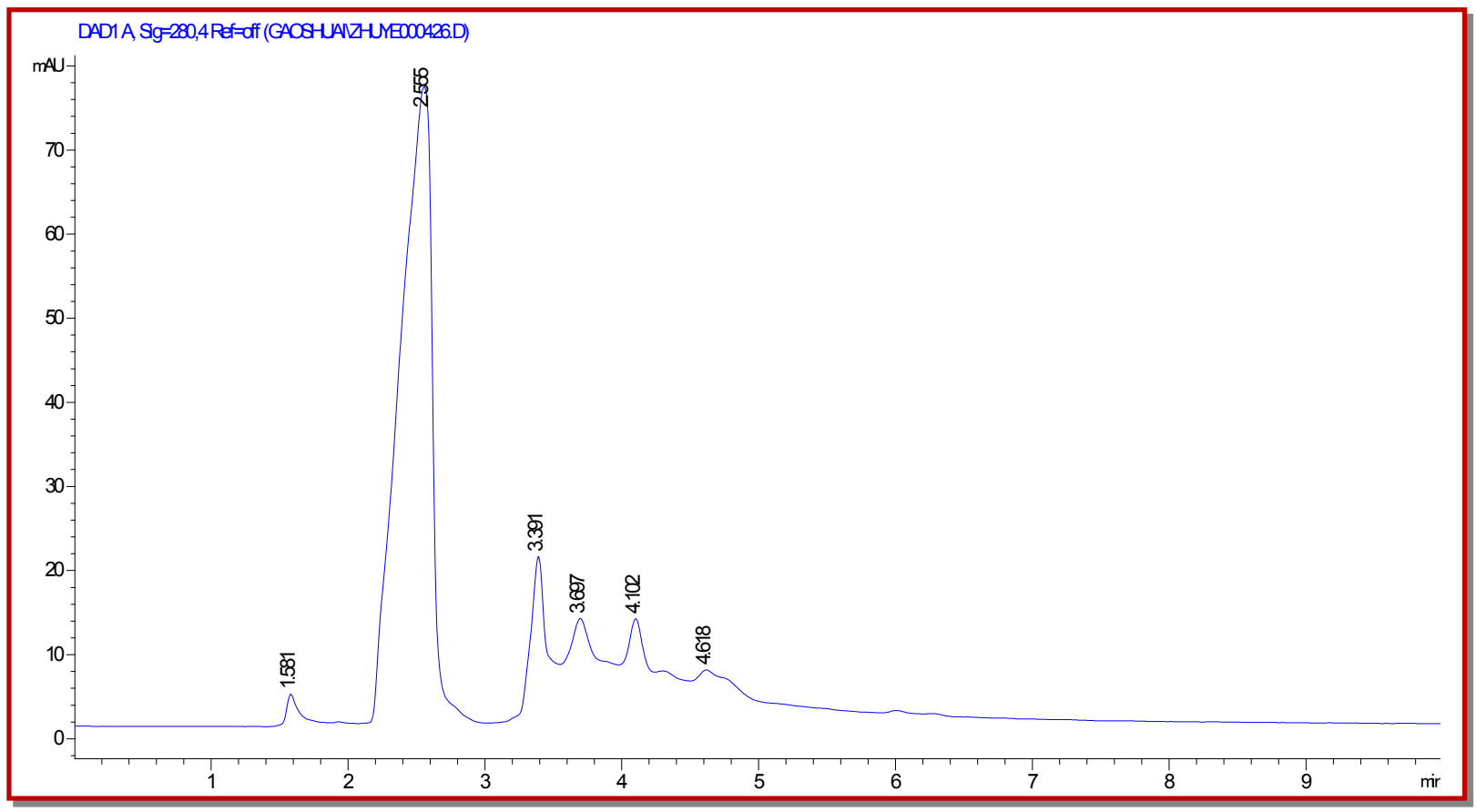

Figure 2: HPLC spectrum of PB2 purified from apple pomace

and subsequently with $1 \mu \mathrm{g} / \mathrm{mL}$ LPS for 20 hours. Meanwhile, effects of pre-treating time from 2 to 24 hours on COX-2 expression in LPS-induced cells were also evaluated. The cells in the blank control group were left untreated and the others in the LPS group were treated with LPS.

Previous tests suggested that PB2 has inhibitory effect on COX-2 protein expression in LPS-induced RAW264.7 cells. However, there is no report on whether PB2 has repairing function or not when inflammation developed in the LPS-induced cells so far. So, investigation on effects of PB2 derived from apple pomace on COX-2 expression in reverse pretreating way was carried out in this work, which the cells were pretreated with LPS followed by PB2.

After incubation, mouse macrophage cell line RAW 264.7 was harvested using a cell scraper and lysed in lysis buffer [20 mM Tris (pH 7.4), $5 \mathrm{mM}$ EDTA, 0.1\% Triton X-100, and $0.01 \%$ 2-mercaptoethanol]. The lysates were sonicated and centrifuged at $15,000 \mathrm{rpm}$ for 10 $\min$ at $4^{\circ} \mathrm{C}$ to remove insoluble material. The supernatant was transferred to a new tube and stored at $-20^{\circ} \mathrm{C}$ until Western blotting analysis. The total protein concentration was measured by the Bio-Rad protein assay kit (Hercules, USA) using bovine serum albumin as the standard. Samples containing equal amounts of protein concentration were heated at $95^{\circ} \mathrm{C}$ for $5 \mathrm{~min}$, and then separated by $10 \%$ sodium dodecyl sulfatepolyacrylamide gel electrophoresis and electrotransferred to NC membranes at $10 \mathrm{~V}$ for 25 min using a semidry transfer (Bio-Rad Ltd., USA). The membranes were blocked for 2 hours at room temperature with Tris -buffered saline containing 5\% non-fat milk, and were then incubated with anti-COX-2 antibody (diluted $1: 1,000)$ for 4 hours at room temperature and subsequently with horseradish peroxidase-conjugated antigoat secondary antibody (diluted 1:2,500) for 1 hour at room temperature. Peroxidase activity was visualized using an ECL kit and a-tubulin was used as an equal loading control.

\section{Western blotting analysis}

COX-2 protein expression was determined by western blotting analysis performed as described elsewhere (Hwangbo et al., 2009). The intensity of each band was quantified using density analysis software (MetaMorph Imaging System, Meta Imaging Series 4.5, BioVision Technologies, Exton, PA, USA), and the density ratio represented the relative intensity of each band against controls in each experiment.

\section{Statistical analysis}

All data were expressed as means \pm standard deviation (SD) from at least three independent experiments. Statistical significance was analyzed by Student's $t$ test and one-way analysis of variance (ANOVA) using SPSS Version 13.0. Value of $\mathrm{p}<0.05$ was considered to be statistically significant differences.

\section{Results}

PB2 content of the purified subfraction from apple 


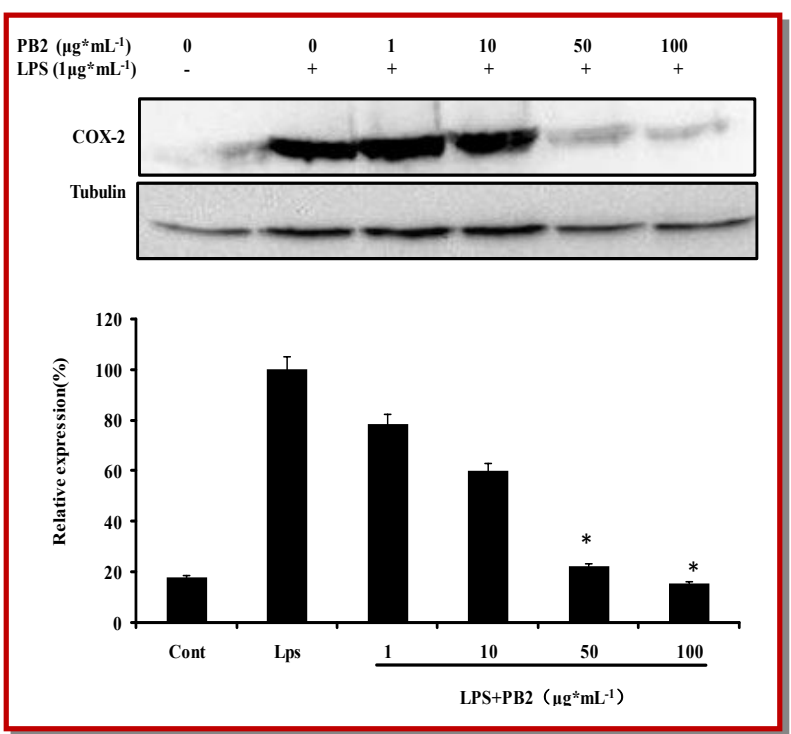

Figure 3: Effect of PB2 with different concentrations on COX-2 expression in LPS-induced RAW 264.7 macrophage cells. *Significant difference: $\mathrm{p}<0.05$ as compared to the LPS-treated group

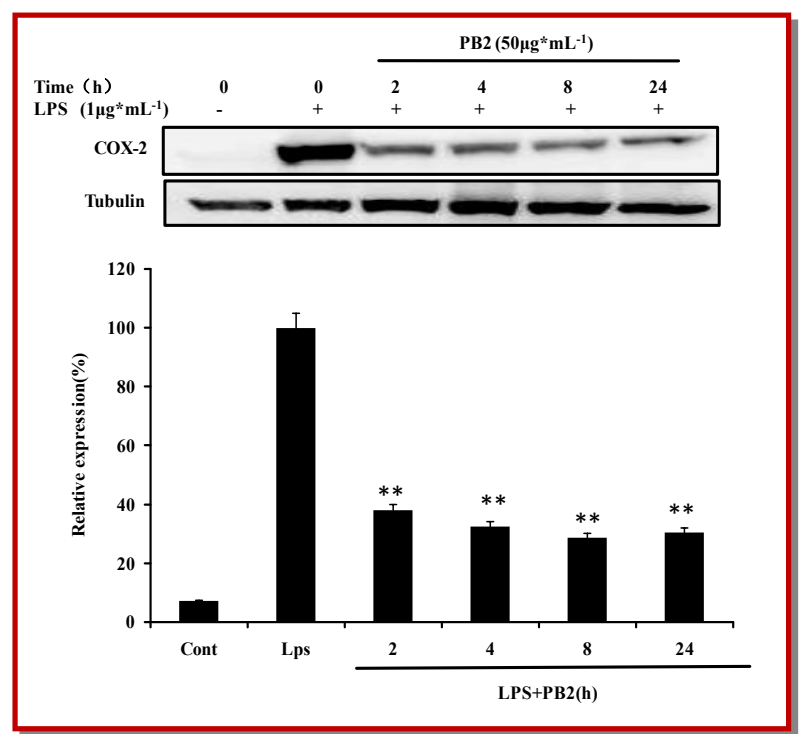

Figure 4: Effect of pretreating time of PB2 on COX-2 expression in LPS-induced RAW 264.7 macrophage cells. *Significant difference: $p<0.05$ as compared to the LPS-treated group

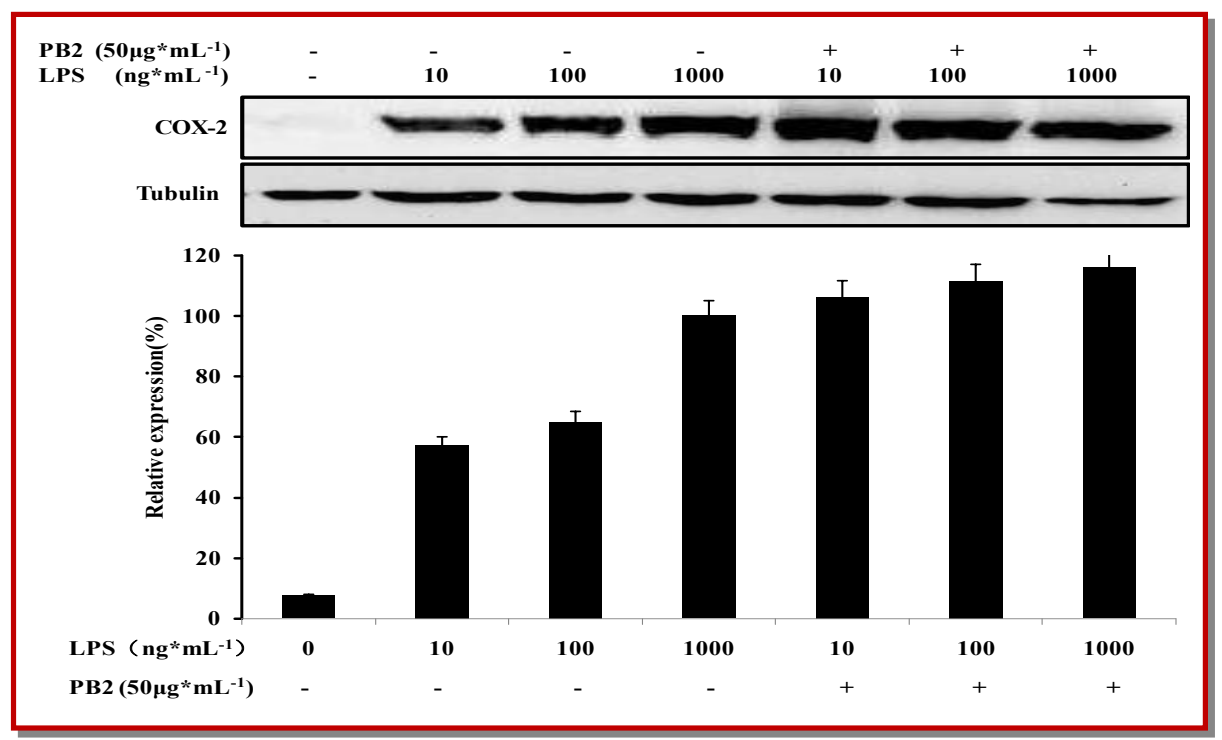

Figure 5: Effects of PB2 in reverse pre-treated way on COX-2 expression in LPS-induced RAW 264.7 macrophage cells

pomace was $72.3 \pm 1.8 \%$, which was increased greatly (Figure 2). It suggested that PB2 with a high purity can be efficiently obtained from apple pomace through fractionation and purification with macroporous absorbent resin and sephadex, respcetively.

Prior to the $1 \mu \mathrm{g} / \mathrm{mL}$ LPS treatment for 20 hours, RAW264.7 cells were pretreated with different concentrations PB2 for 4 hours. Results showed that the expression of COX-2 protein was affected by PB2 (Figure 3). Moreover, the level of COX-2 expression was correlated with PB2 in a concentration-dependent fashion. When the concentration of PB2 reached at least $50 \mu \mathrm{g} / \mathrm{mL}$, the expression of COX-2 protein was signifi- cantly inhibited in LPS-induced cells.

Prior to the $1 \mu \mathrm{g} / \mathrm{mL}$ LPS treatment for 20 hours, RAW264.7 cells were treated with $50 \mu \mathrm{g} / \mathrm{mL}$ PB2 for different hours, respectively. All COX-2 expressions in LPS-induced cells were markedly inhibited by PB2 from 2 to 24 hours (Figure 4). Moreover, the inhibitory activity of PB2 was the strongest at pre-treating time 8 hours.

After being pretreated with different concentration LPS $(10,100,1000 \mathrm{ng} / \mathrm{mL})$ for 20 hours respectively, and then $50 \mu \mathrm{g} / \mathrm{mL}$ PB2 for 4 hours, the express of COX-2 protein were analyzed by Western blotting. The results 
showed that PB2 had no repairing function in LPSinduced cells (Figure 5). The levels of COX-2 expression in the model groups were higher than those in the control groups, which indicated that PB2 could aggravate inflammation in LPS-induced cells. Furthermore, COX-2 protein was expressed in a LPS dose-dependent manner.

\section{Discussion}

PB2 with a high purity of $72.3 \pm 1.9 \%$ was successfully obtained from apple pomace through fractionation and purification using macroporous absorbent resins and sephadex, respectively. Purified PB2 from apple pomace can significantly suppress COX-2 expression at no less than $50 \mu \mathrm{g} / \mathrm{mL}$. And this inhibitory effect was not correlated with pretreating time of PB2 from 2 to 24 hours. However, PB2 has no repairing function in LPSinduced cells. Up to now, these findings have not been reported elsewhere. Our results also suggested that PB2 can be used as an anti-inflammatory nutraceutical for making healthcare product. Apple pomace is one of rich sources of PB2. The recovery of PB2 from apple pomace provides another important biotechnological application of this agro-byproduct.

\section{Acknowledgements}

This work was financially supported by the Natural Science Foundation of China (Project No. 81001381).

\section{References}

Bai XL, Yue TL, Yuan YH, Zhang HW. Optimization of microwave-assisted extraction of polyphenols from apple pomace. J Sep Sci. 2010; 33: 3571-78.

Fujiwara N, Kobayashi K. Macrophages in inflammation. Curr Drug Tar. 2005; 4: 281-86.
García YD, Valles BS, Lobo AP. Phenolic and anti-oxidant composition of by-products from the cider industry: Apple pomace. Food Chem. 2009; 117: 731-38.

Hwangbo C, Lee HS, Park J, Choe J, Lee JH. The antiinflammatory effect of tussilagone, from Tussilago farfara, is mediated by the induction of heme oxygenase- 1 in murine macrophages. Int Immunopharmacol. 2009; 9: 1578-84.

Kim HG, Yoon DH, Kim CH, Shrestha B, Chang WC, Lim SY, Lee WH, Han SG, Lee JO, Lim MH, Kim GY, Choi S, Song WO, Sung JM, Hwang KC, Kim TW. Ethanol extract of Inonotus obliquus inhibits lipopolysaccharide-induced inflammation in RAW 264.7 macrophage cells. J Med Food. 2007; 10: 80-89.

Miura T, Chiba M, Kasai K, Nozaka H, Nakamura T, Shoji T, Kanda T, Ohtake Y, Sato T. Apple procyandins induce tumor cell apoptosis through mitochondrial pathway activation of caspase-3. Carcinogenesis 2008; 29: 585-93.

Sakano K, Mizutani M, Murata M, Oikawa S, Hiraku Y, Kawanishi S. Procyanidin B2 has anti- and pro-oxidant effects on metal-mediated DNA damage. Free Radical Biol Med. 2005; 39: 1041-49.

Suarez B, Alvarez AL, García YD, del Barrio G, Lobo AP, Parra F. Phenolic profiles, anti-oxidant activity and in vitro antiviral properties of apple pomace. Food Chem. 2010; 120: $339-42$.

Vendruscolo F, Albuquerque PCM, Streit F, Esposito E, Ninow JL. Apple pomace: A versatile substrate for biotechnological applications. Crit Rev Biotechnol. 2008; 28: $1-12$.

Wu TT, Tsai CW, Yao HT, Lii CK, Chen HW, Wu YL, Chen PY, Liu KL. Suppressive effects of extracts from the aerial part of Coriandrum sativum L. on LPS-induced inflammatory responses in murine RAW264.7 macrophages. J Sci Food Agric. 2010; 90: 1846-54.

Zhang WY, Liu HQ, Xie KQ, Yin LL, Yu LI, Kwik-Uribe CL, Zhu XZ. Procyanidin dimer B2 [epicatechin-(4 $\beta-8)-$ epicatechin] suppresses the expression of cyclooxygenase-2 in endotoxin-treated monocytic cells. Biochem Bioph Res Commun. 2006; 345: 508-15. 